\title{
Localized surface plate modes via flexural Mie resonances
}

\author{
M. Farhat,,${ }^{1, *}$ P.-Y. Chen, ${ }^{2}$ S. Guenneau, ${ }^{3}$ K. N. Salama, ${ }^{4}$ and H. Bağc1 ${ }^{4}$ \\ ${ }^{1}$ Qatar Environment and Energy Research Institute, Hamad Bin Khalifa University, Qatar Foundation, Doha, Qatar \\ ${ }^{2}$ Department of Electrical and Computer Engineering, Wayne State University, Detroit, Michigan 48202, USA \\ ${ }^{3}$ Aix-Marseille Université, Centre National de la Recherche Scientifique, Centrale Marseille, Institut Fresnel, Campus Universitaire \\ de Saint-Jérôme, 13013 Marseille, France \\ ${ }^{4}$ Division of Computer, Electrical, and Mathematical Science and Engineering, King Abdullah University of Science and Technology, \\ Thuwal 23955-6900, Saudi Arabia \\ (Received 12 January 2017; revised manuscript received 18 April 2017; published 10 May 2017)
}

\begin{abstract}
Surface-plasmon polaritons are naturally generated upon excitation of metals with high-frequency electromagnetic waves. However, the concept of spoof plasmons has made it possible to generate plasmoniclike effects in microwave electrodynamics, magnetics, and even acoustics. Similarly, in this paper, the concept of localized surface plate modes (SPMs) is introduced. It is demonstrated that SPMs can be generated on a two-dimensional (clamped or stress-free) cylindrical surface with subwavelength corrugations, which resides on a thin elastic plate, under excitation by an incident flexural plane wave. Numerical characterization of this corrugated rigid structure shows that it is elastically equivalent to a cylindrical scatterer with dispersive but uniformly negative flexural rigidity. This, indeed, suggests that plasmoniclike elastic materials can be engineered with potential applications in various areas including earthquake sensing and elastic imaging and cloaking.
\end{abstract}

DOI: 10.1103/PhysRevB.95.174201

\section{INTRODUCTION}

Recently, there has been an increasing interest in designing acoustic and elastic metamaterials [1,2]. This research area was practically started by Liu et al., who experimentally demonstrated that (effective) dynamic mass density and bulk modulus may be obtained using locally resonant sonic materials [3]. However, unlike plasmonic metamaterials that rely on (localized or propagating) surface-plasmon polaritons (SPPs) to generate the desired dispersion characteristics, acoustic and elastic metamaterials almost exclusively rely on the geometrical properties of their meta-atoms. The reason is that acoustic and elastic SPPs do not exist in nature [4-6]. As a result, design of such metamaterials has made use of mostly phononic or thin plate crystals [7,8] and resonant cavities or pipes [9-15]. This hindered development of elastic and acoustic metamaterials' applications, which could benefit from plasmoniclike features.

In 2004, Pendry et al. [16] proposed the concept of spoof plasmons. These surface waves or modes act like SPPs but are generated on corrugated metal structures at microwave frequencies, the part of the spectrum where electromagnetic fields cannot penetrate metal surfaces $[16,17]$. The fundamental idea behind the spoof plasmons is the use of periodic subwavelength corrugations to permit surface-bound propagation with a dispersion relation similar to that of the SPPs. Consequently, it can be shown that the corrugated metal structure is equivalent to a uniform structure with material properties, which can be approximated by the Drude metal with a plasma frequency that depends only on the shape and dimensions of the corrugations. This is the analog of surface plasmons in diffraction gratings and their inner relation to Wood's anomaly [18-20]. Pendry's work was almost immediately followed by the experimental demonstration of spoof plasmons at microwave frequencies

\footnotetext{
*mfarhat@hbku.edu.qa
}

[21] and several other studies focusing on various structures capable of supporting spoof plasmons [22-26]. Additionally, localized versions of the spoof plasmons have been demonstrated at microwave frequencies with many intriguing applications including sensing and field enhancement [27,28].

Along the same lines, it has also been shown that surface acoustic waves (SAWs), can be generated on acoustically rigid surfaces with subwavelength corrugations [4,29-31]. The SAWs behave in a way similar to spoof plasmons (and SPPs) and have analogous applications, e.g., their dispersion relation lies outside the acoustic cone and they can be used for subresolution acoustic imaging or sensing. It should be noted here that the acoustic metamaterial filling up the structure, which is equivalent to the corrugated rigid surface, possesses negative effective density [5,32-34]. Additionally, the concept of localized SAWs has been also developed and tested using corrugated rigid cylinders [6].

In this paper, a fourth-order biharmonic wave equation with appropriate boundary conditions, which describes behavior of flexural waves on thin plates, is derived from the generalized elasticity theory [35,37] and is then used in designing an anisotropic flexural metamaterial [38-43]. First, scattering from a cylindrical inhomogeneity under an elastic plane-wave excitation (harmonic vibration of the plate in the vertical direction) is studied. It is shown that in the quasistatic limit the scattering is dominated by the zeroth-order multipole, unlike in the electrodynamics case where the first significant order is the dipolar one. It should be noted here that this is not the only difference between the two scenarios: The fourth-order biharmonic wave equation, which describes the propagation of bending waves in ultrathin plates, is not equivalent to the vector or scalar wave equations, which describe electromagnetic or acoustic wave propagation. Consequently, this paper introduces Mie resonant modes and relevant physics by studying the solution of the fourth-order biharmonic wave equation. Additionally, scattering from a corrugated cylinder in a thin plate with rigid or stress-free grooves is analyzed. 


\section{SETUP OF THE GOVERNING BIHARMONIC EQUATION}

Consider a plate with constant thickness $h$. Let $\rho$ and $D=$ $E h^{3} /\left[12\left(1-v^{2}\right)\right]$ represent the density and flexural rigidity on this plate. Here, $E$ is the Young modulus and $v$ is the Poisson ratio. The plate is excited with a time harmonic source with angular frequency $\omega$. Under the Kirchhoff approximation, it is assumed that $\beta h \ll 1$, i.e., shear deformation and rotary inertia are both negligible in comparison to pure bending $[35,36]$. Here, $\beta$ is the flexural wave number that satisfies $\beta^{4}=$ $\omega^{2} \rho h / D$. Let the vertical displacement (in the $z$ direction), which is generated on the plate upon excitation, be represented by $\zeta(r, \phi)$. In this setup, outside the source region, $\zeta$ satisfies [35-37]

$$
\Delta^{2} \zeta(r, \phi)-\beta^{4} \zeta(r, \phi)=0,
$$

where $\Delta$ represents the Laplacian operator (to be expressed in cylindrical coordinates).

\section{CONDITIONS OF FLEXURAL RESONANT SCATTERING}

Consider the thin plate structure shown in Fig. 1(a). The background plate with rigidity $D_{0}$ and density $\rho_{0}$ includes a circular scatterer with rigidity $D_{\text {in }}$ and density $\rho_{\text {in }}$. It is assumed that the scatterer is centered at the origin $(r=0)$ and its radius is $a$. The excitation is a plane wave propagating in the $x$ direction with displacement field $\zeta^{\mathrm{inc}}(r, \phi)=e^{i \beta_{0} r \cos \phi}$. $\zeta^{\text {inc }}(r, \phi)$ and $\zeta(r, \phi)$, which represents the total displacement field induced on the plate upon excitation, satisfy Eq. (1). Let $\zeta^{\text {scat }}(r, \phi)=\zeta(r, \phi)-\zeta^{\text {inc }}(r, \phi)$ represent the displacement field scattered from the scatterer. Similarly, $\zeta^{\text {scat }}(r, \phi)$ satisfies Eq. (1). To facilitate the solution of Eq. (1) for this setup, $\zeta^{\text {inc }}(r, \phi)$ is expanded as

$$
\zeta^{\mathrm{inc}}(r, \phi)=\sum_{l=0}^{\infty} \varepsilon_{l} i^{l} J_{l}\left(\beta_{0} r\right) \cos (l \phi),
$$

where $\beta_{0}$ is the wave number on the background plate, $\varepsilon_{0}=$ $1, \varepsilon_{l} \geqslant 1=2$, and $J_{l}($.$) are the cylindrical Bessel functions.$ Similar expansions can be used for $\zeta^{\text {scat }}(r, \phi)$ and $\zeta^{\text {in }}(r, \phi)$ as described next. $\zeta^{\text {scat }}(r, \phi)$ must satisfy the radiation condition at $r \rightarrow \infty$, which leads to the expansion

$$
\zeta^{\text {scat }}(r>a, \phi)=\sum_{l=0}^{\infty} i^{l}\left[A_{l} H_{l}^{(1)}\left(\beta_{0} r\right)+B_{l} K_{l}\left(\beta_{0} r\right)\right] \cos (l \phi),
$$
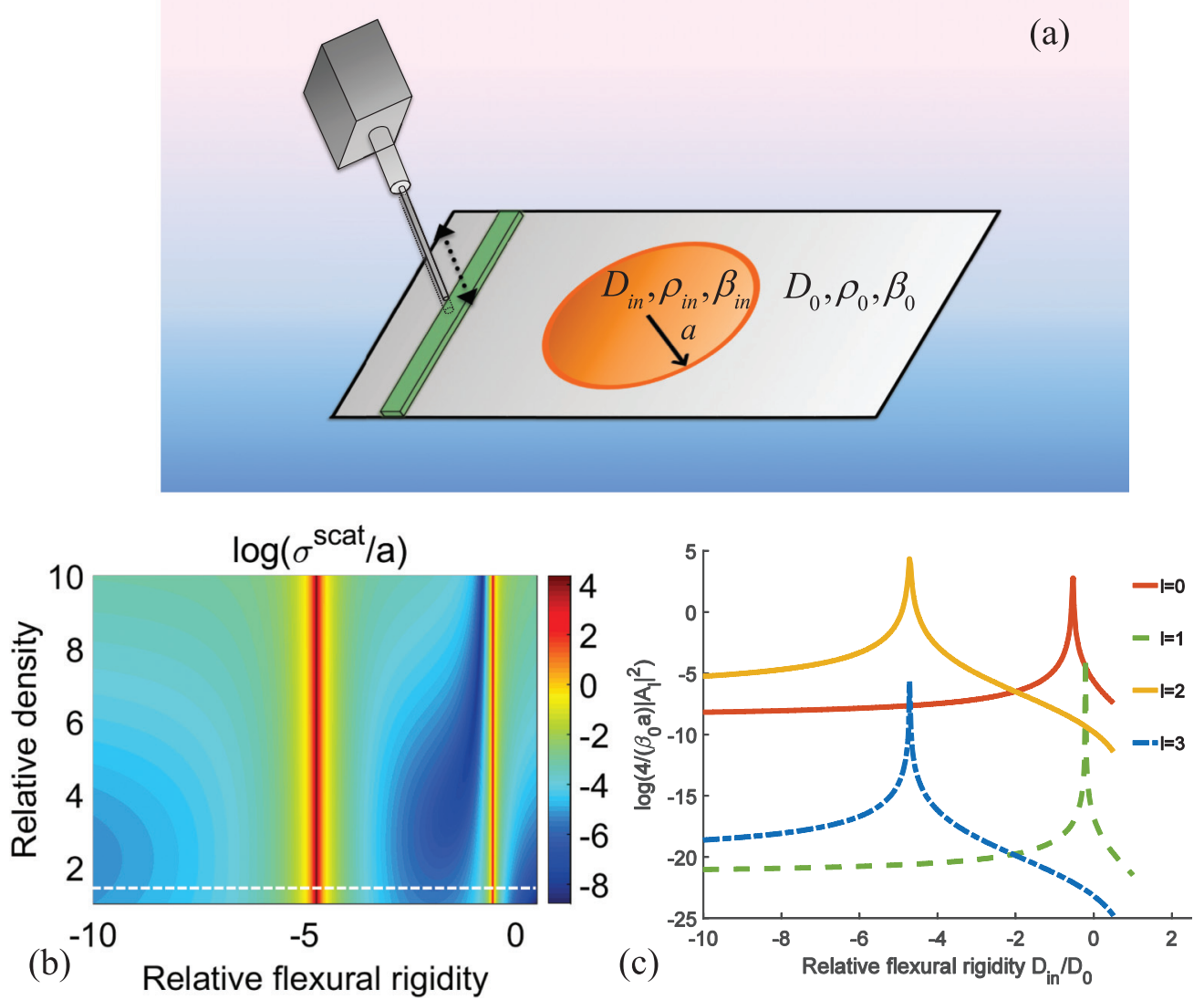

FIG. 1. (a) Sketch of the scattering problem. (b) Normalized scattering cross section $\sigma^{\text {scat }} / a$ in logarithmic scale, of the cylinder vs its relative density $\tilde{\rho}_{i n}=\rho_{i n} / \rho_{0}$ and relative flexural rigidity $\tilde{D}_{i n}=D_{i n} / D_{0}$ for the normalized flexural wave number $\beta_{0} a=0.1$ and Poisson's ratio $v=0.3$. (c) Normalized scattering multipoles $(l=0,1,2,3)$ of the cylindrical inhomogeneity in logarithmic scale for the same wave number and for $\tilde{\rho}_{i n}=1.5$, showing that the fundamental $(l=0)$ and the second-order $(l=2)$ multipole are orders of magnitude higher than the remaining ones. 
outside the scatterer $(r>a)$. Here, $H_{l}^{(1)}($.$) and K_{l}($.$) are the$ cylindrical Hankel functions of the first kind and modified Bessel functions of the second kind, respectively, and $A_{l}$ and $B_{l}$ are the expansion coefficients to be solved for. Inside the scatterer $(r<a), \zeta^{\text {in }}(r, \phi)$ has to be finite at every point, which leads to the expansion

$$
\zeta^{\text {in }}(r, \phi)=\sum_{l=0}^{\infty} i^{l}\left[C_{l} J_{l}\left(\beta_{\text {in }} r\right)+C_{l}^{\prime} I_{l}\left(\beta_{\text {in }} r\right)\right] \cos (l \phi) .
$$

Here, $\beta_{\text {in }}$ is the wave number in the scatterer and $I_{l}($.$) are$ the modified Bessel functions of the first kind, and $C_{l}$ and $C_{l}^{\prime}$ are the expansion coefficients to be solved for. The four sets of unknown coefficients $A_{l}, B_{l}, C_{l}$, and $C_{l}^{\prime}$ are obtained by applying boundary conditions requiring (i) $\zeta$, (ii) its normal derivative $\partial_{r} \zeta$, (iii) the normal component of the bending momentum

$$
M_{r}=-D_{i}\left[\frac{\partial^{2} \zeta}{\partial r^{2}}+v\left(\frac{1}{r} \frac{\partial \zeta}{\partial r}+\frac{1}{r^{2}} \frac{\partial^{2} \zeta}{\partial \phi^{2}}\right)\right],
$$

and (iv) the normal component of the generalized Kirchhoff stress

$$
V_{r}=-D_{i} \frac{\partial(\Delta \zeta)}{\partial r}-D_{i}(1-v) \frac{1}{r^{2}} \frac{\partial}{\partial \phi}\left(\frac{\partial^{2} \zeta}{\partial r \partial \phi}-\frac{1}{r} \frac{\partial \zeta}{\partial \phi}\right)
$$

to be continuous across the boundary at $r=a$. Note that in Eqs. (5) and (6) subscript $i \in\{$ in, 0$\}$, i.e., $D_{i}$, represents either $D_{\text {in }}$ or $D_{0}$ depending on the domain where $M_{r}$ or $V_{r}$ is evaluated. Enforcing these boundary conditions, where $\zeta^{\text {inc }}(r, \phi), \zeta^{\text {scat }}(r, \phi)$, and $\zeta^{\text {in }}(r, \phi)$ are replaced by their appropriate expansions from Eqs. (2)-(4), yields the following linear system for every order $l$ :

$$
\left[\begin{array}{cccc}
H_{l}^{(1)}\left(\beta_{0} a\right) & K_{l}\left(\beta_{0} a\right) & -J_{l}\left(\beta_{\text {in }} a\right) & -I_{l}\left(\beta_{\text {in }} a\right) \\
\beta_{0} H_{l}^{(1)^{\prime}}\left(\beta_{0} a\right) & \beta_{0} K_{l}^{\prime}\left(\beta_{0} a\right) & -\beta_{\text {in }} J_{l}^{\prime}\left(\beta_{\text {in }} a\right) & -\beta_{\text {in }} I_{l}^{\prime}\left(\beta_{\text {in }} a\right) \\
S_{H}\left(\beta_{0} a\right) & S_{K}\left(\beta_{0} a\right) & -S_{J}\left(\beta_{\text {in }} a\right) & -S_{I}\left(\beta_{\text {in }} a\right) \\
T_{H}\left(\beta_{0} a\right) & T_{K}\left(\beta_{0} a\right) & -T_{J}\left(\beta_{\text {in }} a\right) & -T_{I}\left(\beta_{\text {in }} a\right)
\end{array}\right]\left[\begin{array}{c}
A_{l} \\
B_{l} \\
C_{l} \\
C_{l}^{\prime}
\end{array}\right]=-\left[\begin{array}{c}
J_{l}\left(\beta_{0} a\right) \\
\beta_{0} J_{l}^{\prime}\left(\beta_{0} a\right) \\
S_{J}\left(\beta_{0} a\right) \\
T_{J}\left(\beta_{0} a\right)
\end{array}\right] .
$$

Here

$$
\begin{aligned}
& S_{Z}\left(\beta_{i} r\right)=D_{i}\left[l^{2}(1-v) \mp\left(\beta_{i} r\right)^{2}\right] Z_{l}\left(\beta_{i} r\right)-D_{i}(1-v) \beta_{i} r Z_{l}^{\prime}\left(\beta_{i} r\right), \\
& T_{Z}\left(\beta_{i} r\right)=D_{i}\left[l^{2}(1-v)\right] Z_{l}\left(\beta_{i} r\right)-D_{i}\left[l^{2}(1-v) \mp\left(\beta_{i} r\right)^{2}\right] \beta_{i} r Z_{l}^{\prime}\left(\beta_{i} r\right),
\end{aligned}
$$

where the subscript $i \in\{$ in, 0$\}$ as before, and the upper (minus) sign and the lower (plus) sign in the expressions of $S_{Z}$ and $T_{Z}$ refer to $Z_{l}(.) \in\left\{H_{l}^{(1)}(),. J_{l}(),. Y_{l}().\right\}$ and $Z_{l}(.) \in\left\{K_{l}(),. I_{l}().\right\}$, respectively. In the far field, as $r \rightarrow \infty$, the modified Bessel functions $K_{l}\left(\beta_{0} r\right)$ decay exponentially. This means that $\zeta^{\text {scat }}(r>a, \phi)$ consists of only the terms $A_{l} H_{l}^{(1)}\left(\beta_{0} r\right)$ [see Eq. (3)]. In addition, if one considers subwavelength scatterers, i.e., scatterers satisfying $\beta_{0} a \ll 1$, only the first few of these terms contribute to $\zeta^{\text {scat }}(r>a, \phi)$. Indeed, solving Eq. (7) for $l=0,1,2,3 \ldots$, under the conditions $\beta_{\{0, \text { in }\}} a \ll 1$, yields

$$
\begin{aligned}
A_{0}= & -i \frac{\pi}{8} \frac{\tilde{D}_{\text {in }}\left(2-\tilde{\rho}_{\text {in }}\right)-\left[\left(2-\tilde{\rho}_{\text {in }}\right) v+\tilde{\rho}_{\text {in }}\right] /(1+v)}{\tilde{D}_{\text {in }}+(1-v) /(1+v)} \\
& \times\left(\beta_{0} a\right)^{2}+O\left[\left(\beta_{0} a\right)^{3}\right], \\
A_{1}= & -i \frac{\pi}{64} \frac{\tilde{D}_{\text {in }}\left(2-\tilde{\rho}_{\text {in }}\right)-\left[\tilde{\rho}_{\text {in }}+2+\left(2-\tilde{\rho}_{\text {in }}\right) v\right] /(3+v)}{\tilde{D}_{\text {in }}+(1-v) /(3+v)} \\
& \times\left(\beta_{0} a\right)^{4}+O\left[\left(\beta_{0} a\right)^{5}\right], \\
A_{2}= & -i \frac{\pi}{8} \frac{\tilde{D}_{\text {in }}-1}{\tilde{D}_{\text {in }}+(3+v) /(1-v)}\left(\beta_{0} a\right)^{2}+O\left[\left(\beta_{0} a\right)^{3}\right], \\
A_{l}= & o\left[\left(\beta_{0} a\right)^{2}\right], \quad l=3,4 \ldots
\end{aligned}
$$

Here, $\tilde{D}_{\text {in }}=D_{\text {in }} / D_{0}$ and $\tilde{\rho}_{\text {in }}=\rho_{\text {in }} / \rho_{0}$ represent the relative flexural rigidity and relative density, with respect to the surrounding medium, respectively, whereas $o($.) and $O($.) represent the Landau notations [35]. It should be mentioned here that intriguing phenomena, such as negative effective mass density (taking place when the acceleration is out of phase with the dynamic pressure gradient) and negative bulk modulus (occurring when the volume variation becomes out of phase with the dynamic pressure) have been already reported in the framework of elastodynamics. For thin plates, $\tilde{D}_{\text {in }}$ and $\tilde{\rho}_{\text {in }}$ may also take negative values close to resonance $[44,45]$. From the expressions in Eq. (9), one can immediately see that, in contrast to electromagnetic and acoustic scattering, the first-order scattering coefficient $A_{1}$ decays faster than the zeroth- and second-order coefficients, $A_{0}$ and $A_{2}$, as the size of the scatterer is decreased. Additionally, the dependence of $A_{0}$ and $A_{2}$ on the size of the scatterer is of the same order. Similar behavior was already shown in Refs. $[37,39,40]$ for cylindrical hole scatterers in the low-frequency regime. In fact, flexural waves satisfy the fourth-order biharmonic equation while acoustics and electromagnetic waves satisfy the Helmholtz equation. These two equations model two different physical dynamics. A striking example in flexural waves concerns rigid obstacles in the long-wavelength regime where scattering becomes even infinite. For holes in thin plates, the situation is similar to soft cylindrical heterogeneities (first-order negligible in comparison to zeroth and second order). This fact was highlighted in Ref. [37]. However, it is difficult to provide an intuitive explanation of this behavior, in the general case. Indeed, the case of thin plates is an asymptotic limit taken in the full Navier system when the third dimension is much smaller than the other two [46]. As a result, the second-order vector Navier partial differential equations (PDEs) give rise to fourth-order scalar Kirchhoff-Love (KL) PDEs. Navier equations are supplied with continuity of displacement field and stress at each interface (two conditions for second-order PDEs), whereas fourth-order KL equations require continuity of out-of-plane displacement, its normal 
derivative, the normal component of the bending momentum, and the so-called normal component of generalized Kirchhoff stress. One can hardly draw analogies between the case of transverse acoustic and electromagnetic waves propagating in a heterogeneous medium invariant along the third dimension, with the case of heterogeneous thin plates. Therefore, an intuitive interpretation of the dominant monopolar term typical of a clamped inclusion in plates is rather difficult, although one notes that periodically pinned plates display zero-frequency stop bands akin to low-frequency stop bands associated with plasmons in doubly periodic arrays of highly conducting thin and infinite cylinders [45]. Ignoring the terms scaling with $O\left(\beta_{0} a\right)^{l}, l \geqslant 3$, in Eq. (9) one can rewrite the expressions of the dominant terms $A_{0}$ and $A_{2}$ as

$$
\begin{aligned}
& A_{0} \approx-i \frac{\pi}{8}\left(\tilde{D}_{\text {in }}-1\right) \frac{h_{0}\left(\tilde{D}_{\text {in }}, \tilde{\rho}_{\text {in }}\right)}{\tilde{D}_{\text {in }}+f_{0}(v)}\left(\beta_{0} a\right)^{2}, \\
& A_{2} \approx-i \frac{\pi}{8}\left(\tilde{D}_{\text {in }}-1\right) \frac{1}{\tilde{D}_{\text {in }}+f_{2}(\nu)}\left(\beta_{0} a\right)^{2},
\end{aligned}
$$

with $f_{0}(v)=(1-v) /(1+v)$ and $f_{2}(v)=(3+v) /(1-v)$ two positive functions of Poisson's ratio $v$ that satisfies the condition $-1<v \leqslant 0.5$ [35], and $h_{0}$ is a function of $\tilde{\rho}_{\text {in }}$ and $\tilde{D}_{\text {in }}$. In the case of a relative density $\tilde{\rho}_{\text {in }}=1$, i.e., only the flexural rigidity is varying, one has $h_{0}=1$.

Similarly electromagnetic and acoustic waves, one can define a scattering cross-section (SCS) function for flexural waves [37]. Let $g(\phi)=\sqrt{2 r} e^{-i\left(\beta_{0} r-\pi / 4\right)} \lim _{r \rightarrow \infty} \zeta^{\text {scat }}(r, \phi)$ represent the far-field scattering amplitude, which can be used as a measure of scattering strength in the direction $\phi$.

The SCS is then computed by integrating $g(\phi)$ over all angles: $\sigma^{\text {scat }}=\int_{0}^{2 \pi} g(\phi) d \phi$. Inserting Eq. (3) into the expression of $g(\phi)$, taking the limit as $r \rightarrow \infty$, and integrating the result yield

$$
\sigma^{\text {scat }}=\frac{4}{\beta_{0}} \sum_{l=0}^{\infty} \varepsilon_{l}\left|A_{l}\right|^{2} .
$$

It should be noted here that contribution from terms $B_{l} K_{l}\left(\beta_{0} r\right)$ to $\sigma^{\text {scat }}$ vanishes since $K_{l}\left(\beta_{0} r\right)$ exponentially decays to zero as $r \rightarrow \infty$. Generally speaking, the amplitude of $\sigma^{\text {scat }}$ is a measure of an object's detectability by an observer located in the far-field region. For example, in cloaking applications [39], minimizing $\sigma^{\text {scat }}$ would ensure that the object may not be seen by an observer located in the far field irrespective of its relative position with respect to the object. The focus of this paper is to demonstrate the presence of Mie resonances in scattering from elastic structures [see Fig. 1(a)]. These resonances correspond to frequencies where $\sigma^{\text {scat }}$ has a local maximum. Figure 1(b) plots contours of the normalized SCS $\sigma^{\text {scat }} / a$ (in logarithmic scale) of a scatterer with $\beta_{0} a=0.1, v=0.3$, and varying values of $\tilde{\rho}_{\text {in }}$ and $\tilde{D}_{\text {in. }}$. This plot clearly shows that the SCS has two resonances (marked with dark red color) corresponding to negative values of $\tilde{D}_{\text {in }},-0.54$ and -4.71 . These values are predicted by the zeros of the expressions in the denominator in Eq. (10): The first resonance is associated with $A_{0}$, which is given by $\tilde{D}_{i n}=-f_{0}(v)=-(1-v) /(1+v)$, and the second one is associated with $A_{2}$, which is given by $\tilde{D}_{i n}=-f_{2}(v)=$ $-(3+v) /(1-v)$. It should be noted here that these two relations are counterparts of the Fröhlich condition associated
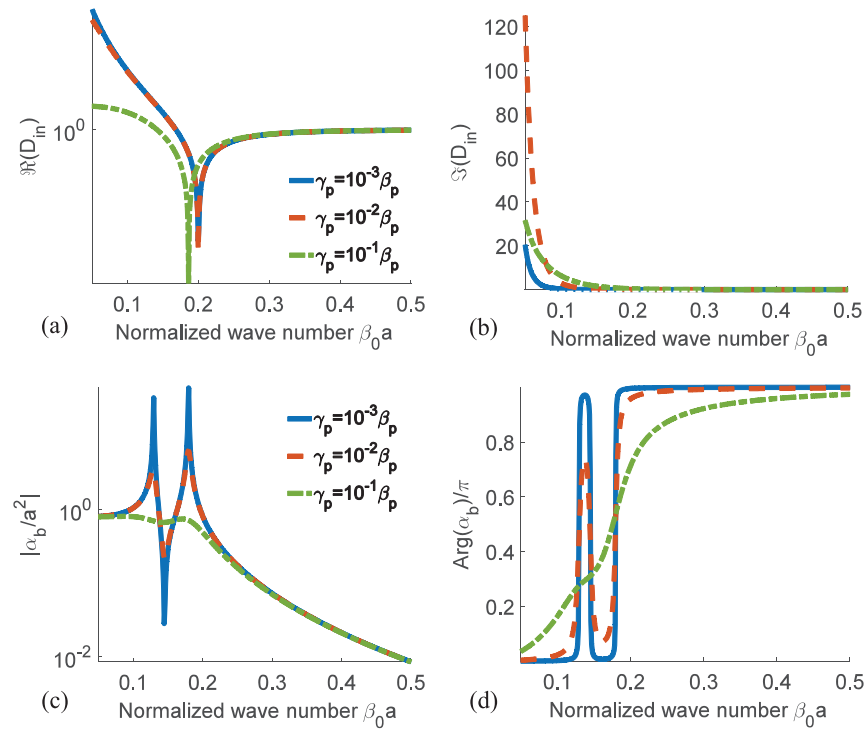

FIG. 2. (a) Real part of the dispersive relative flexural rigidity $D_{i n}$ in logarithmic scale. (b) Imaginary part of $D_{i n}$. (c) Amplitude of the equivalent of normalized polarizability $\alpha_{b} / a^{2}$ in logarithmic scale for the corresponding flexural rigidity given in Fig. 2(a) and for relative density $\tilde{\rho}_{i n}=1$. (d) Phase of $\alpha_{b}$ for the same parameters.

with the electromagnetic dipole surface plasmon [47]. On the other hand, the dependence of the SCS on $\tilde{\rho}_{\text {in }}$ is not as strong and there are no associated resonances in the range considered for $\tilde{\rho}_{\text {in }}$. Figure $1(\mathrm{c})$ plots the normalized scattering coefficients $4 \varepsilon_{l}\left|A_{l}\right|^{2} /\left(\beta_{0} a\right), l=0,1,2,3$ (in logarithmic scale) for the same scatterer with $\beta_{0} a=0.1, v=0.3, \tilde{\rho}_{\text {in }}=1.5$, and varying values of $\tilde{D}_{\text {in }}$ [corresponding to the dashed white curve in Fig. 1(b)]. This figure clearly shows that $A_{0}$ and $A_{2}$ are orders of magnitudes larger than $A_{1}$ and $A_{3}$. This behavior is also predicted correctly by Eq. (9).

Next, another cylindrical scatterer with radius $a$, relative density $\tilde{\rho}_{i n}=1$, and relative flexural rigidity, which satisfies the dispersion function

$$
\tilde{D}_{\text {in }}=1-\beta_{p}^{4} /\left[\beta_{0}^{2}\left(\beta_{0}^{2}+i \gamma_{p}\right)\right],
$$

is considered. In this scattering scenario, $a$ and $\beta_{p}$ satisfy the condition $\beta_{p} a=0.2$, and finally $\gamma_{p}$ represents the loss factor. Real and imaginary parts of $\tilde{D}_{\text {in }}$ (in logarithmic scale) are plotted in Figs. 2(a) and 2(b) for varying values of $\beta_{0} a$ for three different values of $\gamma_{p}$. Particular patterns, which are generated due to the fourth-order dependence on frequency, are observed. In electromagnetics, the polarizability of a scatterer relates its response to the incident field. More specifically, it provides a measure for the strength of the dipole moment induced in the scatterer for a given level of incident electric field. One can define and derive a similar quantity for flexural waves. Let $\alpha_{b}$ represent this physical parameter; $\alpha_{b}$ should account for the terms with coefficients $A_{0}$ and $A_{2}$ (but not only the dipolar mode as in electromagnetics). Consequently, for subwavelength scatterers, $\alpha_{b}$ can be expressed as

$$
\alpha_{b}=\frac{\pi a^{2}}{8}\left(\tilde{D}_{\text {in }}-1\right)\left[\frac{1}{\tilde{D}_{\text {in }}+f_{0}(v)}+\frac{1}{\tilde{D}_{\text {in }}+f_{2}(v)}\right] .
$$



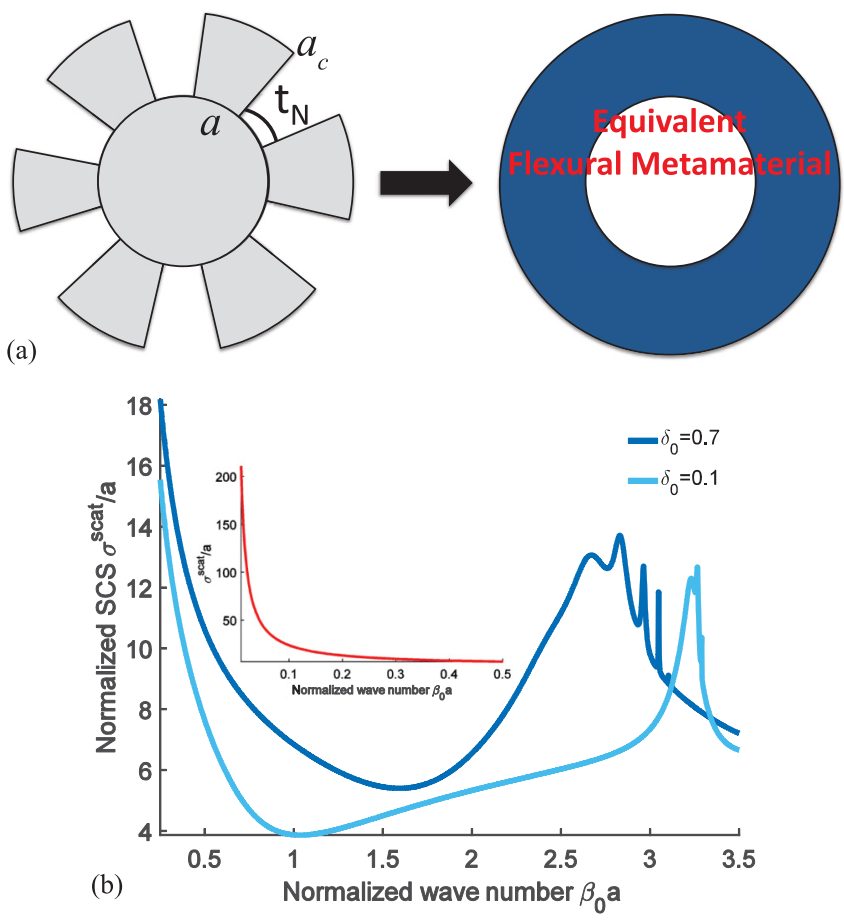

FIG. 3. (a) Equivalent flexural metamaterial model (right) for the corrugated (left) elastic cylinder (with grooves of angle $\theta_{N}=\pi / N$ ). (b) Normalized scattering cross section $\sigma^{\text {scat }} / a$ of the structure shown in (a), versus the normalized wave number. The inset in (b) shows the scattering cross section of a rigid cylinder of same radius showing resonant scattering for $\beta_{0} a \approx 0$ and no resonant effects for higher frequencies.

Note that $\alpha_{b}$ has the unit of a surface. This is because the flexural scattering problem is of two-dimensional nature as opposed to electromagnetic and acoustic scattering problems that are of three-dimensional nature, which leads to an electrical polarizability with the unit of a volume. Figures 2(c) and 2(d) plot the absolute value (in logarithmic scale) and phase of $\alpha_{b}$ for varying values of $\beta_{0} a$ for three different values of $\gamma_{b}$. As expected, $\alpha_{b}$ exhibits a resonance behavior similar to that of $\sigma^{\text {scat }}$. It experiences a resonant enhancement when the denominators $\left|\tilde{D}_{\text {in }}\left(\beta_{0}\right)+f_{0}(v)\right|$ or $\left|\tilde{D}_{\text {in }}\left(\beta_{0}\right)+f_{2}(v)\right|$ in Eq. (13) are minimum. This condition is satisfied at two wave numbers $\beta_{p} /\left[1+f_{0}(v)\right]^{1 / 4}$ and $\beta_{p} /\left[1+f_{2}(v)\right]^{1 / 4}$, showing that the resonance frequencies depend on $\beta_{p}$, a medium parameter that is used in the definition of the dispersion relation for $\tilde{D}_{\text {in }}$. Additionally, as expected, Fig. 2(c) shows that resonant enhancement is lower for higher values of $\gamma_{b}$ [Fig. 2(c)], because the denominator does not completely vanish $\left(\operatorname{Im}\left[\tilde{D}_{\text {in }}\right] \neq 0\right)$ when $\gamma_{b}$ is nonzero. The phase jump of $\pi$ around the resonance wave numbers, shown in Fig. 2(d), confirms the resonant features of the flexural object.

\section{SETUP OF THE SCATTERING PROBLEM OF CORRUGATED STRUCTURES}

Figure 3(a) shows the corrugated cylinder analyzed in this section. It consists of an inner cylinder with radius $a$, outer cylinder with radius $a_{c}$, and periodic grooves with sector angles $\theta_{N}=(2 \pi) /(2 N)=\pi / N$ [Fig. 3(a)]. The material filling the grooves is considered to be the same as the material of the background thin plate and the boundary conditions at the circumference of the corrugated structure are either rigid (clamped) or stress free [35]. It is assumed that the grooves are subwavelength, i.e., $\beta_{0}\left(\pi a_{c} / N\right) \ll 1$, where $\pi a_{c} / N$ is the period of the corrugations. Under this condition, only the fundamental waveguide mode exists inside the grooves [17]. Consequently, one can express the displacement field inside the grooves (for $a \leqslant r \leqslant a_{c}$ ) as

$$
\begin{aligned}
\zeta^{\text {in }}(r, \phi)= & \sum_{l=0}^{\infty} \varepsilon_{l} i^{l}\left[C_{l} Y_{0}\left(\beta_{0} r\right)+C_{l}^{\prime} K_{0}\left(\beta_{0} r\right)\right. \\
& \left.+E_{l} J_{0}\left(\beta_{0} r\right)+F_{l} I_{0}\left(\beta_{0} r\right)\right] \cos (l \phi) .
\end{aligned}
$$

For the internal boundary $r=a$, clamped boundary conditions are used first, that is, $\zeta=\partial \zeta / \partial r=0$. For the external boundary, we use the mode matching technique. In general, this technique relies on matching fields of the electromagnetic, acoustic, and elastic modes present outside the scatterer with those inside the sectors (or grooves) [17]. We use the expansion of the fields in terms of Hankel functions inside and outside the grooves and then apply the matching boundary conditions, i.e., we take into account the corrugations by averaging the physical parameters appearing in the continuity relations, i.e., density for acoustics [33], permittivity and permeability for electromagnetics [27], and flexural rigidity for flexural waves [48]. Thus, for $r=a_{c}$ matching boundary conditions are applied, that is, continuity of all the parameters defined in Sec. III, i.e., $\zeta, \partial_{r} \zeta, M_{r}$, and $V_{r}$ assuming a modified effective flexural rigidity $D_{\text {in }}=\delta D_{0}$ inside the shell $\left(a<r<a_{c}\right)$ shown in Fig. 3(a), taking into account the filling of the grooves [16]. Here $\delta$ is the filling factor of the structure. Applying the six boundary conditions for the system depicted in Fig. 3(a), with the remaining fields in other domains having similar expressions as in the previous section, for each azimuthal order $l$, we obtain a matrix system of equations. In particular, for the scattering unknowns $A_{l}=\psi_{l} / \chi_{l}$ and $B_{l}=\xi_{l} / \chi_{l}$,

$$
\psi_{l}=\left|\begin{array}{cccccc}
-J_{l}\left(\beta_{0} a_{c}\right) & K_{l}\left(\beta_{0} a_{c}\right) & -Y_{0}\left(\beta_{0} a_{c}\right) & -K_{0}\left(\beta_{0} a_{c}\right) & -J_{0}\left(\beta_{0} a_{c}\right) & -I_{0}\left(\beta_{0} a_{c}\right) \\
0 & 0 & Y_{0}\left(\beta_{0} a\right) & K_{0}\left(\beta_{0} a\right) & J_{0}\left(\beta_{0} a\right) & I_{0}\left(\beta_{0} a\right) \\
-\beta_{0} J_{l}^{\prime}\left(\beta_{0} a_{c}\right) & \beta_{0} K_{l}^{\prime}\left(\beta_{0} a_{c}\right) & -\beta_{0} Y_{0}^{\prime}\left(\beta_{0} a_{c}\right) & -\beta_{0} K_{0}^{\prime}\left(\beta_{0} a_{c}\right) & -\beta_{0} J_{0}^{\prime}\left(\beta_{0} a_{c}\right) & -\beta_{0} I_{0}^{\prime}\left(\beta_{0} a_{c}\right) \\
0 & 0 & \beta_{0} Y_{0}^{\prime}\left(\beta_{0} a\right) & \beta_{0} K_{0}^{\prime}\left(\beta_{0} a\right) & \beta_{0} J_{0}^{\prime}\left(\beta_{0} a\right) & \beta_{0} I_{0}^{\prime}\left(\beta_{0} a\right) \\
-S_{J}\left(\beta_{0} a_{c}\right) & S_{K}\left(\beta_{0} a_{c}\right) & -\delta S_{Y}\left(\beta_{0} a_{c}\right) & -\delta S_{K}\left(\beta_{0} a_{c}\right) & \delta S_{J}\left(\beta_{0} a_{c}\right) & \delta S_{I}\left(\beta_{0} a_{c}\right) \\
-T_{J}\left(\beta_{0} a_{c}\right) & T_{K}\left(\beta_{0} a_{c}\right) & -\delta T_{Y}\left(\beta_{0} a_{c}\right) & -\delta T_{K}\left(\beta_{0} a_{c}\right) & \delta T_{J}\left(\beta_{0} a_{c}\right) & \delta T_{I}\left(\beta_{0} a_{c}\right)
\end{array}\right|,
$$




$$
\chi_{l}=\left|\begin{array}{cccccc}
H_{l}^{(1)}\left(\beta_{0} a_{c}\right) & K_{l}\left(\beta_{0} a_{c}\right) & -Y_{0}\left(\beta_{0} a_{c}\right) & -K_{0}\left(\beta_{0} a_{c}\right) & -J_{0}\left(\beta_{0} a_{c}\right) & -I_{0}\left(\beta_{0} a_{c}\right) \\
0 & 0 & Y_{0}\left(\beta_{0} a\right) & K_{0}\left(\beta_{0} a\right) & J_{0}\left(\beta_{0} a\right) & I_{0}\left(\beta_{0} a\right) \\
\beta_{0} H_{l}^{(1)^{\prime}}\left(\beta_{0} a_{c}\right) & \beta_{0} K_{l}^{\prime}\left(\beta_{0} a_{c}\right) & -\beta_{0} Y_{0}^{\prime}\left(\beta_{0} a_{c}\right) & -\beta_{0} K_{0}^{\prime}\left(\beta_{0} a_{c}\right) & -\beta_{0} J_{0}^{\prime}\left(\beta_{0} a_{c}\right) & -\beta_{0} I_{0}^{\prime}\left(\beta_{0} a_{c}\right) \\
0 & 0 & \beta_{0} Y_{0}^{\prime}\left(\beta_{0} a\right) & \beta_{0} K_{0}^{\prime}\left(\beta_{0} a\right) & \beta_{0} J_{0}^{\prime}\left(\beta_{0} a\right) & \beta_{0} I_{0}^{\prime}\left(\beta_{0} a\right) \\
S_{H}\left(\beta_{0} a_{c}\right) & S_{K}\left(\beta_{0} a_{c}\right) & -\delta S_{Y}\left(\beta_{0} a_{c}\right) & -\delta S_{K}\left(\beta_{0} a_{c}\right) & \delta S_{J}\left(\beta_{0} a_{c}\right) & \delta S_{I}\left(\beta_{0} a_{c}\right) \\
T_{H}\left(\beta_{0} a_{c}\right) & T_{K}\left(\beta_{0} a_{c}\right) & -\delta T_{Y}\left(\beta_{0} a_{c}\right) & -\delta T_{K}\left(\beta_{0} a_{c}\right) & \delta T_{J}\left(\beta_{0} a_{c}\right) & \delta T_{I}\left(\beta_{0} a_{c}\right)
\end{array}\right|,
$$

and

$$
\xi_{l}=\left|\begin{array}{cccccc}
H_{l}^{(1)}\left(\beta_{0} a_{c}\right) & -J_{l}\left(\beta_{0} a_{c}\right) & -Y_{0}\left(\beta_{0} a_{c}\right) & -K_{0}\left(\beta_{0} a_{c}\right) & -J_{0}\left(\beta_{0} a_{c}\right) & -I_{0}\left(\beta_{0} a_{c}\right) \\
0 & 0 & Y_{0}\left(\beta_{0} a\right) & K_{0}\left(\beta_{0} a\right) & J_{0}\left(\beta_{0} a\right) & I_{0}\left(\beta_{0} a\right) \\
\beta_{0} H_{l}^{(1)^{\prime}}\left(\beta_{0} a_{c}\right) & -\beta_{0} J_{l}^{\prime}\left(\beta_{0} a_{c}\right) & -\beta_{0} Y_{0}^{\prime}\left(\beta_{0} a_{c}\right) & -\beta_{0} K_{0}^{\prime}\left(\beta_{0} a_{c}\right) & -\beta_{0} J_{0}^{\prime}\left(\beta_{0} a_{c}\right) & -\beta_{0} I_{0}^{\prime}\left(\beta_{0} a_{c}\right) \\
0 & 0 & \beta_{0} Y_{0}^{\prime}\left(\beta_{0} a\right) & \beta_{0} K_{0}^{\prime}\left(\beta_{0} a\right) & \beta_{0} J_{0}^{\prime}\left(\beta_{0} a\right) & \beta_{0} I_{0}^{\prime}\left(\beta_{0} a\right) \\
S_{H}\left(\beta_{0} a_{c}\right) & -S_{J}\left(\beta_{0} a_{c}\right) & -\delta S_{Y}\left(\beta_{0} a_{c}\right) & -\delta S_{K}\left(\beta_{0} a_{c}\right) & \delta S_{J}\left(\beta_{0} a_{c}\right) & \delta S_{I}\left(\beta_{0} a_{c}\right) \\
T_{H}\left(\beta_{0} a_{c}\right) & -T_{J}\left(\beta_{0} a_{c}\right) & -\delta T_{Y}\left(\beta_{0} a_{c}\right) & -\delta T_{K}\left(\beta_{0} a_{c}\right) & \delta T_{J}\left(\beta_{0} a_{c}\right) & \delta T_{I}\left(\beta_{0} a_{c}\right)
\end{array}\right|,
$$

with the same notations for the functionals $S_{Z}$ and $T_{Z}$ given in Eq. (9), with the main difference here being that for columns 3-6 only Bessel or Hankel functions of order $l=0$ are taken into account, due to the subwavelength nature of the corrugations [Eq. (15)] and the presence of the extra term $\delta=\theta_{1} /\left(\theta_{1}+\theta_{2}\right)$, accounting for the filling factor of the corrugations $(\delta=0.5$ if $\left.\theta_{1}=\theta_{2}\right)$. The other possibility with flexural waves is to ensure stress-free boundary conditions at $r=a$, that is, $M_{r}=V_{r}=0$. Applying the six boundary conditions for the system, for each azimuthal order $l$, we obtain again an algebraic system of equations, in particular for the scattering unknowns $A_{l}=\psi_{l} / \chi_{l}$ and $B_{l}=\xi_{l} / \chi_{l}$ (here we keep the same notations for the scattering coefficients for simplicity):

$$
\begin{aligned}
& \psi_{l}=\left|\begin{array}{cccccc}
-J_{l}\left(\beta_{0} a_{c}\right) & K_{l}\left(\beta_{0} a_{c}\right) & -Y_{0}\left(\beta_{0} a_{c}\right) & -K_{0}\left(\beta_{0} a_{c}\right) & -J_{0}\left(\beta_{0} a_{c}\right) & -I_{0}\left(\beta_{0} a_{c}\right) \\
-\beta_{0} J_{l}^{\prime}\left(\beta_{0} a_{c}\right) & \beta_{0} K_{l}^{\prime}\left(\beta_{0} a_{c}\right) & -\beta_{0} Y_{0}^{\prime}\left(\beta_{0} a_{c}\right) & -\beta_{0} K_{0}^{\prime}\left(\beta_{0} a_{c}\right) & -\beta_{0} J_{0}^{\prime}\left(\beta_{0} a_{c}\right) & -\beta_{0} I_{0}^{\prime}\left(\beta_{0} a_{c}\right) \\
-S_{J}\left(\beta_{0} a_{c}\right) & S_{K}\left(\beta_{0} a_{c}\right) & -\delta S_{Y}\left(\beta_{0} a_{c}\right) & -\delta S_{K}\left(\beta_{0} a_{c}\right) & \delta S_{J}\left(\beta_{0} a_{c}\right) & \delta S_{I}\left(\beta_{0} a_{c}\right) \\
0 & 0 & \delta S_{Y}\left(\beta_{0} a\right) & \delta S_{K}\left(\beta_{0} a\right) & \delta S_{J}\left(\beta_{0} a\right) & \delta S_{I}\left(\beta_{0} a\right) \\
-T_{J}\left(\beta_{0} a_{c}\right) & S_{K}\left(\beta_{0} a_{c}\right) & -\delta T_{Y}\left(\beta_{0} a_{c}\right) & -\delta T_{K}\left(\beta_{0} a_{c}\right) & \delta T_{J}\left(\beta_{0} a_{c}\right) & \delta T_{I}\left(\beta_{0} a_{c}\right) \\
0 & 0 & \delta T_{Y}\left(\beta_{0} a\right) & \delta T_{K}\left(\beta_{0} a\right) & \delta T_{J}\left(\beta_{0} a\right) & \delta T_{I}\left(\beta_{0} a\right)
\end{array}\right|, \\
& \chi_{l}=\left|\begin{array}{cccccc}
H_{l}^{(1)}\left(\beta_{0} a_{c}\right) & K_{l}\left(\beta_{0} a_{c}\right) & -Y_{0}\left(\beta_{0} a_{c}\right) & -K_{0}\left(\beta_{0} a_{c}\right) & -J_{0}\left(\beta_{0} a_{c}\right) & -I_{0}\left(\beta_{0} a_{c}\right) \\
\beta_{0} H_{l}^{(1)^{\prime}}\left(\beta_{0} a_{c}\right) & \beta_{0} K_{l}^{\prime}\left(\beta_{0} a_{c}\right) & -\beta_{0} Y_{0}^{\prime}\left(\beta_{0} a_{c}\right) & -\beta_{0} K_{0}^{\prime}\left(\beta_{0} a_{c}\right) & -\beta_{0} J_{0}^{\prime}\left(\beta_{0} a_{c}\right) & -\beta_{0} I_{0}^{\prime}\left(\beta_{0} a_{c}\right) \\
S_{H}\left(\beta_{0} a_{c}\right) & S_{K}\left(\beta_{0} a_{c}\right) & -\delta S_{Y}\left(\beta_{0} a_{c}\right) & -\delta S_{K}\left(\beta_{0} a_{c}\right) & \delta S_{J}\left(\beta_{0} a_{c}\right) & \delta S_{I}\left(\beta_{0} a_{c}\right) \\
0 & 0 & \delta S_{Y}\left(\beta_{0} a\right) & \delta S_{K}\left(\beta_{0} a\right) & \delta S_{J}\left(\beta_{0} a\right) & \delta S_{I}\left(\beta_{0} a\right) \\
T_{H}\left(\beta_{0} a_{c}\right) & S_{K}\left(\beta_{0} a_{c}\right) & -\delta T_{Y}\left(\beta_{0} a_{c}\right) & -\delta T_{K}\left(\beta_{0} a_{c}\right) & \delta T_{J}\left(\beta_{0} a_{c}\right) & \delta T_{I}\left(\beta_{0} a_{c}\right) \\
0 & 0 & \delta T_{Y}\left(\beta_{0} a\right) & \delta T_{K}\left(\beta_{0} a\right) & \delta T_{J}\left(\beta_{0} a\right) & \delta T_{I}\left(\beta_{0} a\right)
\end{array}\right|,
\end{aligned}
$$

and

$$
\xi_{l}=\left|\begin{array}{cccccc}
H_{l}^{(1)}\left(\beta_{0} a_{c}\right) & -J_{l}\left(\beta_{0} a_{c}\right) & -Y_{0}\left(\beta_{0} a_{c}\right) & -K_{0}\left(\beta_{0} a_{c}\right) & -J_{0}\left(\beta_{0} a_{c}\right) & -I_{0}\left(\beta_{0} a_{c}\right) \\
\beta_{0} H_{l}^{(1)^{\prime}}\left(\beta_{0} a_{c}\right) & -\beta_{0} J_{l}^{\prime}\left(\beta_{0} a_{c}\right) & -\beta_{0} Y_{0}^{\prime}\left(\beta_{0} a_{c}\right) & -\beta_{0} K_{0}^{\prime}\left(\beta_{0} a_{c}\right) & -\beta_{0} J_{0}^{\prime}\left(\beta_{0} a_{c}\right) & -\beta_{0} I_{0}^{\prime}\left(\beta_{0} a_{c}\right) \\
S_{H}\left(\beta_{0} a_{c}\right) & -S_{J}\left(\beta_{0} a_{c}\right) & -\delta S_{Y}\left(\beta_{0} a_{c}\right) & -\delta S_{K}\left(\beta_{0} a_{c}\right) & \delta S_{J}\left(\beta_{0} a_{c}\right) & \delta S_{I}\left(\beta_{0} a_{c}\right) \\
0 & 0 & \delta S_{Y}\left(\beta_{0} a\right) & \delta S_{K}\left(\beta_{0} a\right) & \delta S_{J}\left(\beta_{0} a\right) & \delta S_{I}\left(\beta_{0} a\right) \\
T_{H}\left(\beta_{0} a_{c}\right) & -S_{J}\left(\beta_{0} a_{c}\right) & -\delta T_{Y}\left(\beta_{0} a_{c}\right) & -\delta T_{K}\left(\beta_{0} a_{c}\right) & \delta T_{J}\left(\beta_{0} a_{c}\right) & \delta T_{I}\left(\beta_{0} a_{c}\right) \\
0 & 0 & \delta T_{Y}\left(\beta_{0} a\right) & \delta T_{K}\left(\beta_{0} a\right) & \delta T_{J}\left(\beta_{0} a\right) & \delta T_{I}\left(\beta_{0} a\right)
\end{array}\right| .
$$

\section{LOCALIZED SPMS INDUCED ON CORRUGATED THIN PLATE CYLINDERS}

Next, it is demonstrated that localized surface plate modes (SPMs) can be induced on corrugated thin plate structures. First, consider the case of corrugations with clamped boundary conditions [Fig. 3(a)]. The scattering coefficients $A_{l}$ associated with the corrugated structure are deduced from Eqs. (15)-
(17) and $\sigma^{\text {scat }}$ is computed using Eq. (11). Figure 3(b) plots $\sigma^{\text {scat }} / a$ for varying values of $\beta_{0} a$ between 0.25 and 3 for two different values of $\delta_{0}$. It can be noticed from the figure that $\sigma^{\text {scat }}$ increases as $\beta_{0}$ approaches zero. Indeed, Eq. (11) shows that $\sigma^{\text {scat }}$ grows with $1 / \beta_{0}$ as $\beta_{0}$ approaches zero. This is a wellknown phenomenon: Flexural scattering from rigid objects, which are characterized with conditions $\zeta=0$ and $\partial \zeta / \partial r=0$ 
on their boundaries, is divergent at zero frequency, as seen in the inset of Fig. 3(b) where the SCS from a rigid object with radius $a$ is shown for comparison [35-37,39]. This means that a pin in a thin plate has a natural resonance in the quasistatic regime. Figure 3(b) also shows that, in addition to the zerofrequency resonance, $\sigma^{\text {scat }}$ has many peaks in the higher end of the spectrum, which correspond to the corrugated structure's resonances.

The presence of multiple resonances in the scattering spectrum of this rather simple structure can be explained by carefully looking at how the expressions of $\chi_{l}, \psi_{l}$, and $\xi_{l}$ in Eqs. (15)-(17), which are used to compute the scattering coefficients $A_{l}$ and $B_{l}$ and eventually $\sigma^{\text {scat }}$, are obtained. This is done by comparing the method, which is used for obtaining expressions of the scattering coefficients associated with classical (rigid or stress-free) core-shell structures, to the one used here to obtain Eqs. (15)-(17) [36,37,39,40]. The main difference is the representation of $\zeta$ inside the shell, i.e., only the zeroth-order Bessel and Hankel functions are taken into account in Eq. (14), due to the presence of subwavelength corrugations. Additionally, there is a scaling factor in the expressions of the determinants $\chi_{l}, \psi_{l}$, and $\xi_{l}$ : $\delta=\theta_{1} /\left(\theta_{1}+\theta_{2}\right)$, which accounts for the filling fraction of the grooves. As expected, the resonance peak locations and bandwidths are sensitive to the changes in $\delta$. Figure 3(b) plots $\sigma^{\text {scat }}$ for two values of $\delta, 0.1$ (dilute regime) and 0.7 (high filling). It is clear that bandwidths of the resonances are larger for $\delta=0.7$. Additionally, reducing $\delta$ shifts the resonances towards higher frequencies. It should also be noted here that the resonances at higher frequencies are sensitive to loss. However, if the level of loss is high, one can expect that some of these modes will simply vanish and only the broadband mode (at lower frequencies) will remain.

Next, the same corrugated structure but with stress-free conditions is considered. The scattering coefficients $A_{l}$ associated with this structure are deduced from Eqs. (18)-(20) and $\sigma^{\text {scat }}$ is again computed using Eq. (11). Figure 4(a) plots $\sigma^{\text {scat }} / a$ for varying values of $\beta_{0} a$ between 0 and 3 for $\delta=0.7$. It can be immediately observed that, unlike the corrugated structure with clamped boundary conditions, there is no resonance at the zero frequency. This is expected since static scattering from any stress-free structures is by definition nonresonant [37]. However, introducing corrugations to the scatterer results in generation of higher frequency resonances in the spectrum of $\sigma^{\text {scat }}$. To verify that these peaks are associated with the higher-order modes of the corrugated structure, normalized scattering coefficients $4 /\left(\beta_{0} a\right)\left|A_{l}\right|^{2}, l=0,1, \ldots, 10$ are also plotted in Fig. 4(a). These curves show that each resonant peak of $\sigma^{\text {scat }}$ is associated with a resonance of a scattering coefficient with given order $l$. Unlike for the higher-order scattering coefficients, the zeroth-order coefficient (red line) does not display a sharp resonance associated with it.

Figure 4(c) plots the distribution of the near-field displacement $\zeta$ 's amplitude at $\beta_{0} a=1.3$ and $\beta_{0} a=2.75$. Higherorder nature of the modes is clearly seen.

\section{DISPERSION RELATION OF SPMs}

In this section, we would like to analyze the tunability properties of the corrugated structure of Fig. 3(a) as well as

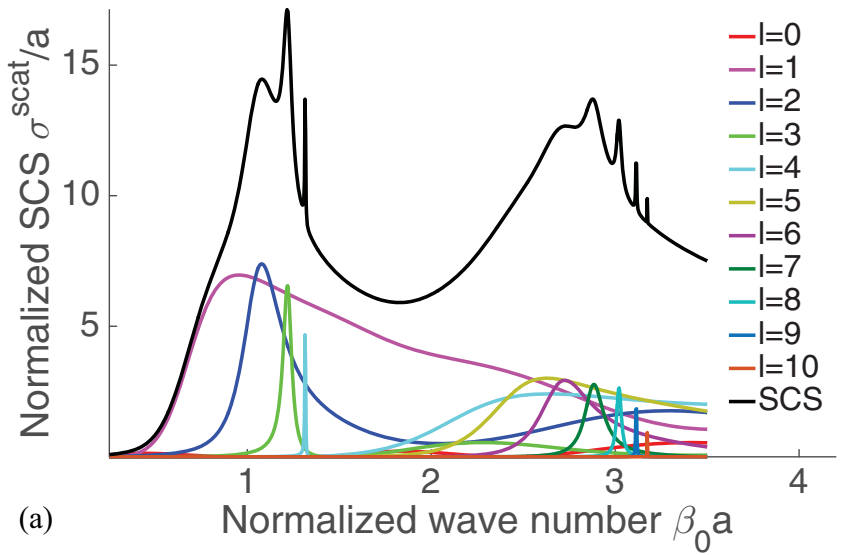

(b)
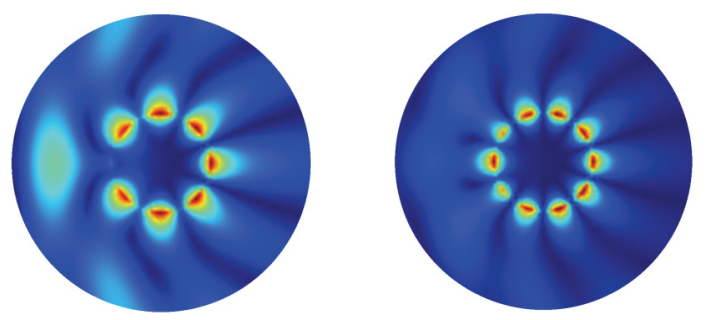

FIG. 4. (a) Normalized scattering cross section $\sigma^{\text {scat }} / a$ of the corrugated cylinder shown in Fig. 3(a) vs the normalized wave number, using stress-free boundary conditions (black curve). The different multipoles $(l=0 \ldots 10)$ corresponding to determinants in Eqs. (18)-(20) are plotted to show the contribution of each multipolar resonance. (b) Near field plots of the displacement $\zeta(r, \phi)$ for normalized wave numbers 1.3 and 2.75 , corresponding to modes $l=4$ and 6 .

the possibility to treat it as an effective metamaterial with homogeneous flexural rigidity as schematized in Fig. 3(a). In order to better understand the behavior of the corrugated cylinder, the dispersion curves of the SPM originating from the one-dimensional equivalent grating of the same geometrical parameters are computed $[6,16]$. The resonant wave number of the equivalent material is taken as $\beta_{p} a=\pi / \sqrt{2}$. The normalized frequency $\left(\beta_{0} a\right)^{2}$ is given versus the normalized propagation constant of the normalized plate spoof plasmon $\beta_{\text {SPM }} a$ in Fig. 5(a) for different loss factors [and zoomed in Fig. 5(c) for low frequencies]. It should be noted here that the dispersion of waves in free space is of parabolic nature and is not linear as is the case for acoustics and electromagnetics. This means that the elastic cone is of parabolic shape (red curve). It can be seen that for lower wave numbers the dispersion relation is identical to the free-space one. For higher wave numbers, the behavior changes and the dispersion of the SPM becomes flat (with convergence to $\sqrt{2} \beta_{p}$ ) and located outside the elastic cone. This behavior is a hallmark of localized surface-plasmon polaritons in optical frequencies and shows undoubtedly that the corrugated cylinder of Fig. 3(a) gives rise to similar features in elasticity (flexural waves in thin plates). Additionally, from the dispersion relations of Fig. 5(b) [zoomed in Fig. 5(d) for low frequencies], one can conclude that, ultimately, the dispersion of SPMs is very sensitive to the environment parameters and therefore can be a platform for elasticity sensors. 

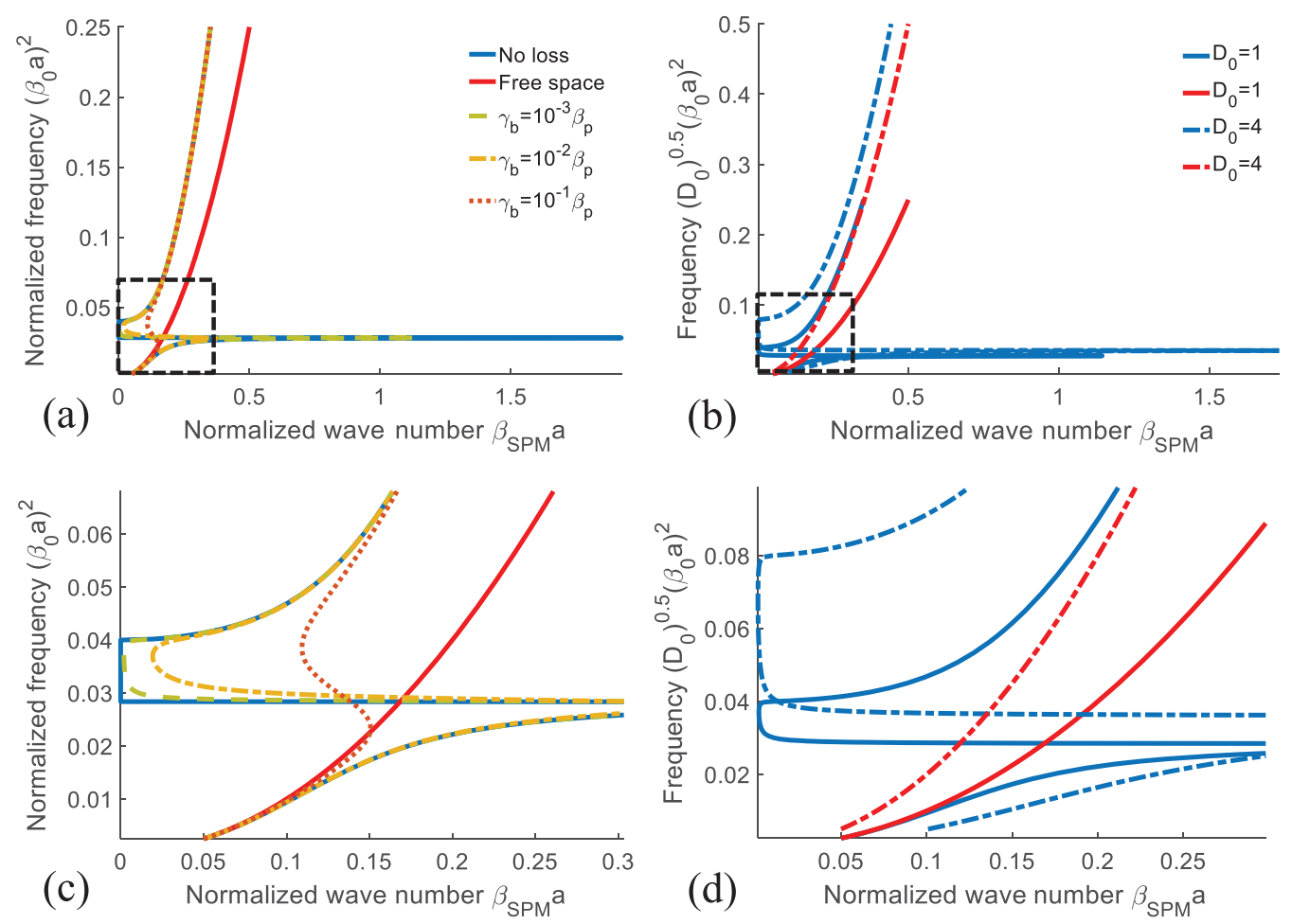

FIG. 5. (a) Dispersion relation of the SPM vs normalized frequency $\left(\beta_{0} a\right)^{2}$ for $D_{0}=1$ and for different values of the loss factor $\gamma_{b}=$ $\left\{0,10^{-3}, 10^{-2}, 10^{-1}\right\} \times \beta_{p}$, with $\beta_{p}$ the natural wave number of the model of Eq. (13). (b) Same as (a) but for different values of the relative flexural rigidity of the surrounding medium $D_{0}=1$ and 4 . (c) and (d) are zoomed versions of the plots of (a) and (b), respectively.

\section{CONCLUSIONS}

We have introduced here the concept and potential realization of an elastic localized surface mode, obtained by a subwavelength corrugation of a rigid or stress-free elastic cylinder in a thin plate. We have demonstrated with analytical analysis that the features of these plasmons are very much similar to their electromagnetic and acoustic counterparts. The experimental realization of this idea [structure of Fig. 3(a)] may be within reach in the near future. Note, for instance, that lensing of bending waves via negative refraction was theoretically predicted using the biharmonic plate model [38] and experimentally confirmed in a thin Duralumium plate [14]). This will allow for exciting applications of interest to elastodynamics, including subwavelength imaging and sensing for the oil and gas industry, elastic wave guiding, and enhancement of nonlinear effects.

\section{ACKNOWLEDGMENTS}

M.F. acknowledges funding by the Qatar National Research Fund through a National Priorities Research Program Exceptional Grant No. NPRP X-107-1-027.

\section{APPENDIX: METHODS}

Analytical methods based on scattering Mie theory of cylindrical objects in thin elastic plates are used to obtain the results in this study. The vertical displacement of the plate is the solution of the fourth-order differential biharmonic KL equation. We proceed, as is usually done, by expanding the impinging plane waves and the scattered fields in terms of Bessel and Hankel functions in the polar coordinate system centered with the object. We then apply proper elastodynamic boundary conditions on each cylindrical interface in order to obtain the scattering coefficients for waves, which uniquely determine the displacement fields everywhere. The displacement field distributions and scattering cross sections are computed using Bessel developments and Eq. (11), respectively. In the quasistatic limit, where the size of the elastic core sphere is much smaller than the wavelength and only the lowest-order Mie coefficient remains important, an analytical formula is obtained which is reminiscent of a pinned inclusion in a plate [Eq. (10)]. We have numerically checked that proper convergence for all the results is reached (via truncation order).
[1] Acoustic Metamaterials: Negative Refraction, Imaging, Lensing and Cloaking, edited by R. V. Craster and S. Guenneau (Springer-Verlag, London, 2012).

[2] Transformation Wave Physics: Electromagnetics, Elastodynamics, and Thermodynamics, edited by M. Farhat, P.-Y. Chen, S. Guenneau, and S. Enoch (Pan Stanford, Singapore, 2016).
[3] Z. Liu et al., Locally resonant sonic materials, Science 289, 1734 (2000).

[4] J. Christensen et al., Collimation of sound assisted by acoustic surface waves, Nat. Phys. 3, 851 (2007).

[5] I. Spiousas, D. Torrent, and J. Sanchez-Dehesa, Experimental realization of broadband tunable resonators based 
on anisotropic metafluids, Appl. Phys. Lett. 98, 244102 (2011).

[6] M. Farhat, P. Y. Chen, and H. Bagci, Localized acoustic surface modes, Appl. Phys. A 122, 377 (2016).

[7] Phononic Crystals: Fundamentals and Applications, edited by A. Khelif and A. Adibi (Springer, New York, 2015).

[8] M. Kadic, T. Bückmann, R. Schittny, and M. Wegener, Metamaterials beyond electromagnetism, Rep. Prog. Phys. 76, 126501 (2013).

[9] S. Yang, J. H. Page, Z. Liu, M. L. Cowan, C. T. Chan, and P. Sheng, Focusing of Sound in a 3D Phononic Crystal, Phys. Rev. Lett. 93, 024301 (2004).

[10] M. Farhat, S. Guenneau, S. Enoch, G. Tayeb, A. B. Movchan, and N. V. Movchan, Analytical and numerical analysis of lensing effect for linear surface water waves through a square array of nearly touching rigid square cylinders, Phys. Rev. E 77, 046308 (2008).

[11] M. Farhat, S. Guenneau, S. Enoch, and A. Movchan, All-anglenegative-refraction and ultra-refraction for liquid surface waves in 2D phononic crystals, J. Comput. Appl. Math. 234, 2011 (2010).

[12] G. Dupont, M. Farhat, A. Diatta, S. Guenneau, and S. Enoch, Numerical analysis of three-dimensional acoustic cloaks and carpets, Wave Motion 48, 483 (2011)

[13] A. B. Movchan, N. B. Movchan, and R. C. McPhedran, BlochFloquet bending waves in perforated thin plates, Proc. R. Soc. A 463, 2505 (2007).

[14] M. Dubois et al., Flat lens for pulse focusing of elastic waves in thin plates, Appl. Phys. Lett. 103, 071915 (2013).

[15] M. Farhat, S. Guenneau, and S. Enoch, Broadband cloaking of bending waves via homogenization of multiply perforated radially symmetric and anisotropic thin elastic plates, Phys. Rev. B 85, 020301 (2012).

[16] J. B. Pendry, Martin-L. Moreno, and F. J. Garcia-Vidal, Mimicking surface plasmons with structured surfaces, Science 305, 847 (2004).

[17] A. F. Harvey, Periodic and guiding structures at microwave frequencies, IRE Trans. Microwave Theory Tech. 8, 30 (1960).

[18] R. W. Wood, On a remarkable case of uneven distribution of light in a diffraction grating spectrum, Philos. Mag. 4, 396 (1902).

[19] Lord Rayleigh, Note on the remarkable case of diffraction spectra described by Prof. Wood, Philos. Mag. 14, 60 (1907).

[20] R. C. McPhedran, and D. Maystre, A detailed theoretical study of the anomalies of a sinusoidal diffraction grating, Opt. Acta 21, 413 (1974).

[21] A. P. Hibbins, B. R. Evans, and J. R. Sambles, Experimental verification of designer surface plasmons, Science 308, 670 (2005).

[22] F. J. Garcia de Abajo and J. J. Saenz, Electromagnetic Surface Modes in Structured Perfect-Conductor Surfaces, Phys. Rev. Lett. 95, 233901 (2005).

[23] F. J. Garcia-Vidal, L. Martin-Moreno, and J. B. Pendry, Surfaces with holes in them: New plasmonic metamaterials, J. Opt. A 7 , S97 (2005).

[24] M. A. Kats, D. Woolf, R. Blanchard, N. Yu, and F. Capasso, Spoof plasmon analogue of metal-insulator-metal waveguides, Opt. Express 19, 14860 (2011).
[25] R. Stanley, Plasmonics in the mid-infrared, Nat. Photon. 6, 409 (2012).

[26] M. Alaoui et al., Cyclic concentrator, carpet cloaks and fisheye lens via transformation plasmonics, J. Opt. 18, 044023 (2016).

[27] A. Pors, E. Moreno, L. Martin-Moreno, J. B. Pendry, and F. J. Garcia-Vidal, Localized Spoof Plasmons Arise while Texturing Closed Surfaces, Phys. Rev. Lett. 108, 223905 (2012).

[28] P. A. Huidobro, X. Shen, J. Cuerda, E. Moreno, L. MartinMoreno, F. J. Garcia-Vidal, T. J. Cui, and J. B. Pendry, Magnetic Localized Surface Plasmons, Phys. Rev. X 4, 021003 (2014).

[29] Z. He, H. Jia, C. Qiu, Y. Ye, R. Hao, M. Ke, and Z. Liu, Nonleaky surface acoustic waves on a textured rigid surface, Phys. Rev. B 83, 132101 (2011).

[30] J. Christensen, Z. Liang, and M. Willatzen, Metadevices for the confinement of sound and broadband double-negativity behavior, Phys. Rev. B 88, 100301(R) (2013).

[31] J. Christensen, Z. Liang, and M. Willatzen, Minimal model for spoof acoustoelastic surface states, AIP Advances 4, 124301 (2014).

[32] M. Ambati, N. Fang, C. Sun, and X. Zhang, Surface resonant states and superlensing in acoustic metamaterials, Phys. Rev. B 75, 195447 (2007).

[33] D. Torrent, and J. Sanchez-Dehesa, Anisotropic Mass Density by Radially Periodic Fluid Structures, Phys. Rev. Lett. 105, 174301 (2010).

[34] R. Gracia-Salgado, V. M. Garcia-Chocano, D. Torrent, and J. Sanchez-Dehesa, Negative mass density and $\rho$-near-zero quasi-two-dimensional metamaterials: Design and applications, Phys. Rev. B 88, 224305 (2013).

[35] S. Timoshenko, Theory of Plates and Shells (McGraw-Hill, New York, 1940).

[36] K. F. Graff, Wave Motion in Elastic Solids (Dover, New York, 1975).

[37] A. N. Norris and C. J. Vemula, Sound Vibr. 181, 115 (1995).

[38] M. Farhat, S. Guenneau, and S. Enoch, High directivity and confinement of flexural waves through ultra-refraction in thin perforated plates, EPL-Europhys. Lett. 91, 54003 (2010).

[39] M. Farhat et al., Platonic scattering cancellation for bending waves in a thin plate, Sci. Rep. 4, 4644 (2014).

[40] D. Torrent, Y. Pennec, and B. Djafari-Rouhani, Effective medium theory for elastic metamaterials in thin elastic plates, Phys. Rev. B 90, 104110 (2014).

[41] M. J. Smith, R. C. McPhedran, C. G. Poulton, and M. H Meylan, Negative refraction and dispersion phenomena in platonic clusters, Waves Random Complex Media 22, 435 (2012).

[42] M. J. A. Smith, M. H. Meylan, and R. C. McPhedran, Flexural wave filtering and platonic polarisers in thin elastic plates, Q. J. Mech. Appl. Math. 66, 437 (2013).

[43] M. J. Smith, R. C. McPhedran, and M. H. Meylan, Double Dirac cones at $\mathrm{k}=0$ in pinned platonic crystals, Waves Random Complex Media 24, 35 (2014).

[44] J. C. Hsu, Low-frequency forbidden bands in phononic crystal plates with Helmholtz resonators, Jap. J. Appl. Phys. 50, 07HB01 (2011). 
[45] T. Antonakakis and R. V. Craster, High-frequency asymptotics for microstructured thin elastic plates and platonics, Proc. R. Soc. A 468, 1408 (2012).

[46] S. A. Nazarov, Asymptotic theory of thin plates and rods, Vol. 1, Reduction of dimension and integral estimates (Nauchanaya Kniga (IDMI), Novosibirck, 2002).
[47] S. A. Maier, Plasmonics: Fundamentals and Applications (Springer, New York, 2007).

[48] A. B. Movchan, N. V. Movchan, and C. G. Poulton, Asymptotic Models of Fields in Dilute and Densely Packed Composites (World Scientific, Singapore, 2002). 